\title{
Developmental origins of health and disease: reducing the burden of chronic disease in the next generation
}

\author{
Peter D Gluckman,2, Mark A Hanson³ and Murray D Mitchell*4
}

\begin{abstract}
Despite a wealth of underpinning experimental support, there has been considerable resistance to the concept that environmental factors acting early in life (usually in fetal life) have profound effects on vulnerability to disease later in life, often in adulthood. This has resulted in an unwillingness among public health decision makers to implement relatively simple approaches, based upon an understanding of developmental plasticity and intergenerational influences, to reducing the burden of disease particularly in low socioeconomic groups.
\end{abstract}

The concept of developmental origins of health and disease is predicated upon the assumption that environmental factors acting early in life (usually in fetal life) have profound effects on vulnerability to disease later in life, often in adulthood. The range of experimental, clinical and epidemiological data linking conditions in early life to later health is now overwhelming [1]. Initially, the focus was on a small fraction of children -those who were born small - but it is now clear that the environment impacts on the development of every child [2]. Observations and experimental approaches have generally considered nutritional changes or, classically, alterations in glucocorticosteroid exposure, reflecting the critical maturational events linked to such events. Indeed, the placenta is in a critical position to cause or modify such challenges by altering nutritional transport functions or the pattern and nature of endocrine signals impacting the fetus. Nor does the story end at birth, because epigenetic development can be influenced by how the infant is fed,

*Correspondence: murray.mitchell@uq.edu.au

${ }^{4} \mathrm{UQ}$ Centre for Clinical Research, Building 71/198, Royal Brisbane and Women's

Hospital Campus, Brisbane, Queensland 4072, Australia

Full list of author information is available at the end of the article and perhaps how its gut is colonized with commensal bacteria.

Yet there has been considerable resistance to these ideas. Medicine is replete with reductionist biomedical thinking and this has, in some ways, limited not only our understanding but also our ability to address the challenge of some contemporary health problems. Nowhere is this clearer than in the outcomes of genomewide association studies where, despite substantial investment, only a relatively small proportion of risk of common non-communicable diseases (NCDs) - such as cardiovascular disease and diabetes - is explained [3]. The economic and humanitarian costs of NCDs are enormous in both the developed and the developing world, and indeed they may destabilize the economies of lowincome countries where recent data show that risk markers for these diseases become evident early in the process of socioeconomic improvement, and well below the level of affluence associated with their appearance in developed societies. From research laboratory to public health department, the focus on the processes that influence risk of NCD has been largely on genetic or adult lifestyle factors, to the exclusion of a third, critical component, namely development. We now recognize that incorporating an understanding of developmental plasticity and intergenerational influences, operating directly or indirectly through epigenetic mechanisms and potentially over more than one generation, is essential to understanding variation in the individual risk of NCD, for example, in an obesogenic environment [4].

Epigenetic processes provide a biological mechanism to explain how environmental influences can affect the phenotypic variation of a generation, or several generations, including their susceptibility to NCDs [4]. These epigenetic processes were formerly thought to be primarily involved in gene dosage regulation via parental imprinting and $\mathrm{X}$ chromosome inactivation, and in regulating cell lineage differentiation. But environmental influences during development, such as maternal diet or endocrine status, can affect offspring phenotype via epigenetic effects on a range of non-imprinted genes, for 
example, the glucocorticoid receptor [5] and nuclear receptors such as the peroxisome proliferator-activated receptors [6]. These processes can operate from before conception into early postnatal life and can affect neuroendocrine and endocrine function, fat and muscle development, and metabolic control processes such as mitochondrial function [4].

Phenotypic outcomes with long-term consequences thus involve the interplay between genetic, developmental and environmental influences. It is impossible to pull them apart. In contrast to the fatalism of a deterministic view, this new synthesis gives room for optimism. The epigenetic state has been shown to be reversible, with persistent effects from transient interventions during a critical developmental window in animal models [6,7] suggesting the potential for early intervention. In cancer biology, epigenetic biomarkers and drug targeting are burgeoning fields but, as yet, little attention has been given to the potential for epigenetic measurements in early-life interventions to reduce the incidence of NCDs. The ways adults interact with the environment, food preference, appetite control, mood and exercise capacity, for example, have been shown experimentally to be influenced by development $[2,6]$. Perhaps then the efficacy of the very interventions that form the mainstay of metabolic and cardiovascular disease prevention in adults is affected by early-life exposures.

If we adopt a developmental perspective, the question arises - what new initiatives might be taken? In adolescent pregnancies the conflict between allocation of nutrients to the woman or to her fetus is usually resolved in favor of the mother. This makes evolutionary sense but the epigenetically induced constraint puts the offspring at greater risk of later disease [2]. Thus, educational and social initiatives aimed at delaying the age of first pregnancy until four years after menarche, to allow maternal somatic and particularly pelvic growth to be completed, would not only empower women in controlling their lives but also promote the health of the next generation. The cultural and political issues preventing implementation of such a strategy need to be confronted.

Similar considerations apply to maternal diet and body composition at conception. Animal studies demonstrate long-term effects of both excessive and deficient nutrition in this period on the offspring, and human data reveal effects on birth outcomes. Studies in the UK show that many women do not eat prudently around conception [8], and only a minority of women becoming pregnant, whether planned or not, adhere to guidelines about a healthy diet and lifestyle [9]. Moreover, gestational obesity and diabetes, themselves with developmental origins, both leave lasting effects on the offspring of the next generation, and so the cycle of risk is perpetuated.
Education and other ways to address these problems need to be explored. Nutrition and workload in pregnancy need greater attention, and not only in the developing world. In Japan, birth weight has been falling in recent years and this is attributed to inadequate weight gain in pregnancy, sometimes following ill-conceived medical advice. Smoking, drug and alcohol abuse, HIVAIDS and malaria still complicate far too many pregnancies. Human development is far from complete at birth, and recent data confirm the importance of exclusive breastfeeding for up to four to six months in promoting optimal growth, resistance to infection, cardiovascular health and neurocognitive development. Yet breastfeeding rates are low even in developed countries such as the UK, and the number of women reaching the goal is far too low - support and encouragement for them are needed urgently.

None of these solutions seems sophisticated, although it may have taken the recent insights into underlying developmental epigenetic mechanisms to emphasize them. But, when viewed in terms of their potential impact, especially in developing societies and in lower socioeconomic groups in developed countries, it is clear that their importance has been underestimated. Public health decision makers should reflect on this and consider possible actions [10]. We fear that eradicating the inflexibility of past biomedical concepts about development, in favor of a softer integrative synthesis, will be seen as too difficult - we may continue to find it easier to think deterministically about chronic disease and then to be surprised when, perhaps because they are made too late, interventions turn out to be relatively ineffective. Adopting a developmental perspective may not influence our approach to minimizing risk of NCDs in today's adults. But surely, when we see potential risk from that angle, we should resolve to use our developmental perspective to devise interventions in time to help the next generation.

Abbreviations

NCD, non-communicable disease.

Competing interests

The authors declare that they have no competing interests.

Authors' contributions

Each author has contributed equally to the final manuscript.

\section{Author details}

'Liggins Institute, The University of Auckland, Private Bag 92019, Auckland 1142 , New Zealand

${ }^{2}$ National Research Centre for Growth and Development, The University of Auckland, Private Bag 92019, Auckland 1142, New Zealand

${ }^{3}$ Centre for Developmental Origins of Health and Disease, University of

Southampton, Southampton SO16 5YA, UK

${ }^{4}$ UQ Centre for Clinical Research, Building 71/198, Royal Brisbane and Women's Hospital Campus, Brisbane, Queensland 4072, Australia

Published: 24 February 2010 


\section{References}

1. Newnham JP, Ross MG (Eds): Early Life Origins of Human Health and Disease. Basel: Karger; 2009

2. Gluckman PD, Hanson MA, Beedle AS: Early life events and their consequences for later disease: a life history and evolutionary perspective. Am J Hum Biol 2007, 19:1-19.

3. Maher B: Personal genomes: the case of the missing heritability. Nature 2008, 456:18-21.

4. Gluckman PD, Hanson MA, Cooper C, Thornburg KL: Effect of in utero and early-life conditions on adult health and disease. N Engl J Med 2008, 359:61-73.

5. Weaver IC, Cervoni N, Champagne FA, D'Alessio AC, Sharma S, Seckl JR, Dymov S, Szyf M, Meaney MJ: Epigenetic programming by maternal behavior. Nat Neurosci 2004, 7:847-854.

6. Gluckman PD, Lillycrop KA, Vickers MH, Pleasants AB, Phillips ES, Beedle AS, Burdge GC, Hanson MA: Metabolic plasticity during mammalian development is directionally dependent on early nutritional status. ProC Natl Acad Sci U S A 2007, 104:12796-12800.
7. Park JH, Stoffers DA, Nicholls RD, Simmons RA: Development of type 2 diabetes following intrauterine growth retardation in rats is associated with progressive epigenetic silencing of Pdx1. J Clin Invest 2008, 118:2316-2324.

8. Robinson SM, Crozier SR, Borland SE, Hammond J, Barker DJP, Inskip HM: Impact of educational attainment on the quality of young women's diets. Eur J Clin Nutr 2004, 58:1174-1180.

9. Inskip HM, Crozier SR, Godfrey KM, Borland SE, Cooper C, Robinson SM Women's compliance with nutrition and lifestyle recommendations before pregnancy: general population cohort study. BMJ 2009, 338:b481.

10. World Health Organization. Promoting Optimal Fetal Development: Report of a Technical Consultation. Geneva: WHO; 2006.

doi:10.1186/gm135

Cite this article as: Gluckman PD, et al:: Developmental origins of health and disease: reducing the burden of chronic disease in the next generation. Genome Medicine 2010, 2:14. 